Konstantinos Drakos *, loannis Malandrakis **

\section{Global Versus Non-Global Banks: A Capital Ratios-Based Analysis}

\begin{abstract}
This paper examines the Leverage Ratio and Total Capital Ratio of global versus non-global banks in both the pre- and postcrisis periods. A panel data set of 165 global and non-global financial institutions from 38 countries is used for the period 1999-2015 and a random effects model is employed to examine whether global banks perform better or not compared to their non-global counterparts. This study comes up with two important findings. First, global banks do not exhibit heterogeneous behaviour with respect to both ratios neither in the pre- and especially nor in the post-crisis period. Second, the Leverage Ratio is crisis-insensitive, but the Total Capital Ratio is not. Our findings encourage further research on the topic of the contribution of global banks to the financial crisis propagation (at least as far as leverage is concerned).
\end{abstract}

Keywords: CAMELS factors; financial crisis; Global banks; Leverage Ratio; Non-global banks; Total Capital Ratio.

JEL classification: C33, G01, G21, G24, G29.

\section{Introduction}

Global banks are important for their investment and lending activities worldwide, but also suspect for contributing to the transmission of financial crises across banking systems and economies, due to their interconnectedness and the degree of their exposure to cross-border funding activities (Kalemli-Ozcan, Papaioannou \& Perri, 2013; Liu \& Pogach, 2017). Additionally, global banks fall
${ }^{*}$ Department of Accounting and Finance, Athens University of Economics and Business,

Athens, Greece

Email:

kdrakos@aueb.gr

${ }^{* *}$ Department of Accounting and Finance, Athens University of Economics and Business, Athens, Greece

Email: imalandrakis@aueb.gr 
into the "too big to fail" category, and their analysis in terms of regulatory ratios etc. still constitutes a hot topic, given that the recent bailouts of large financial institutions (multinational banks mostly) in the U.S. and Europe resulted in governments' balance sheets burden and significant costs to the taxpayers (Moosa, 2010).

This paper explores (a) whether global banks differ from their non-global counterparts in terms of Leverage and Total Capital ratios, in both the pre- and postfinancial crisis periods, and (b) whether a possibly inferior performance of global banks may have contributed to the 2007-2008 crisis. Several CAMELS factors (Non-performing loans, Operational Expenses to Operational Income, ROE, and Loan-to-Deposit ratios) and bank size are employed as explanatory variables. These factors are used for supervisory purposes as well as in comparative banking sectors studies (Beck, Demirgüc-Kunt \& Merrouche, 2013).

The value of this study lies in that it compares global and non-global banks and considers a risk and a non-risk adjusted capital ratio using a broad data set involving both bank types from all continents. The findings are notable concerning existing literature on global banking and risk- versus non-risk weighted capital ratios. First, it is indicated that the 2007-2008 financial crisis has positively affected only the Total Capital Ratio of both bank-types. Second, global banks do not exhibit differential behaviour in terms of both capital ratios in both the preand the post-crisis period. Third, global banks do not present superior or inferior performance relative to non-global banks during and after the recent financial crisis. Finally, it is shown that the traditional Leverage Ratio (of both global and non-global banks) is crisis insensitive.

The remainder of this paper is organized as follows. Section 2 presents related literature. Section 3 describes variables selection and data used and Section 4 analyses estimation methodology and hypotheses being tested. Section 5 presents empirical analysis and discusses the results. Finally, Section 6 concludes.

\section{Related literature}

The global market position of a systemically important financial institution identifies it as a global systematically important bank (G-SIB) or not (Yuksel, 2014). The Basel Committee on Banking Supervision (BCBS) classifies a bank as G-SIB if it fulfils five basic criteria (each one equally weighted at 20\%): size, interconnectedness, substitutability/financial institution infra-structure, global activity, 
and complexity (BCBS, 2013; 2017). G-SIBs and consequently global banks ${ }^{1}$ are subject to higher capital buffer requirements, loss absorbing and recapitalization capacity as well as supervisory expectations (Financial Stability Board - FSB, 2016). The G-SIBs list is annually updated based on the G-SIB assessment methodology and is submitted to the Financial Stability Board for endorsement and publication (BCBS, 2017).

Concerning financial crises propagation and global banks contribution, Cetorelli \& Goldberg (2010) examine global banks contribution to the 2007-2008 financial crisis transmission in emerging economies across Europe, Asia, and Latin America and find that loan supply in these countries was affected significantly by liquidity shortage of both foreign-owned and local banks due to the degree of their exposure to cross-border funding and to the internal capital markets of the broader banking organisation in which they participate. Hale, Kapan \& Minoiu (2016) study the transmission of financial sector shocks across borders through international bank connections, using a large data set of long-term interbank loans for 6,000 banks during the time period 1997-2012, and show, inter alia, that (a) direct exposures to crisis countries significantly reduce banks' profit margins, and indirect exposures to these countries enhance this effect and (b) interconnected financial systems facilitate shocks transmission.

Global banks are sensitive to financial crises; Altunbas, Manganelli \& MarquesIbanez (2011) analyse a large data set of European and U.S. banks focusing on the global financial crisis and show, among other things, that bank size is positively related to bank risk level (probability of distress), a result that is in line with the view that global banks were significantly riskier during the recent financial crisis. In addition, De Haan \& Kakes (2019) study the accumulated losses of both large and small European financial institutions for the period 2007-2016 and find that large banks (including G-SIBs) were particularly hit by the 2007-2008 global financial crisis.

\footnotetext{
1 McCauley, Ruud \& Wooldridge (2002) use the terms "global" and "multinational banks" equivalently and mention that a global bank uses funds raised in a foreign market to finance its claims on borrowers in the same foreign market. The concepts of global bank and G-SIB are not always coinciding; it is possible for a global bank not to be a G-SIB if it does not meet all of the necessary criteria. Also the terms "multinational" and "global" seem to have equal meaning (Niepmann, 2011; De Haas \& Van Lelyveld, 2014), although "global bank" has a broader meaning than "multinational bank": a multinational bank can operate in more than one country within the same region (e.g. Europe) while a global bank can operate in more than one region, excluding parent's institution continent (for instance a European multinational bank in Latin America, Africa, etc.).
} 


\section{Data and variables}

The panel data set covers the period 1999 to 2015 and includes observations (on a yearly basis) of certain CAMELS variables for 165 banks, of which 71 are global and 94 are non-global, from 38 countries worldwide (see Appendix A - Table A1).

As mentioned above, the dependent variables are the Leverage Ratio and the Total Capital Ratio. Leverage Ratio is a non-risk adjusted capital ratio and it seems a good predictor of bank (in-) solvency, covering both crisis and non-crisis periods, since it is a less sensitive ratio to systemic shocks and thus a more objective measure of banks' capital adequacy (see e.g. EBA, 2016; Smith, Grill \& Lang, 2017). In addition, it has been shown that this ratio - at region and country level - exhibits significantly lower procyclical behaviour compared to the Total Capital Ratio (Pfeifer \& Pikhart, 2019; Turguttopbas, 2018)2. Also, Leverage Ratio can be used as a measure to reduce procyclicality of bank lending (Behn, Haselmann $\&$ Wachtel, 2016) ${ }^{3}$. Moreover, the Basel Committee on Banking Supervision put special emphasis on Leverage Ratio of global banks, as it recently published a consultation document concerning minimum buffer requirements for the Leverage Ratio of G-SIBs (see BCBS, 2019). The main risk-based capital adequacy indicator, i.e. Total Capital Ratio, is strongly connected with asset quality and earnings ability (Francis, 2014) and it is a significant risk predictor alongside Leverage Ratio (Hogan, 2015).

As for the explanatory variables, certain bank-specific variables from each CAMELS $^{4}$ segment are used. Non-performing loans (NPLs) ratio (proxy for asset quality) is well documented in the literature since and it has been shown, inter alia, that it is a significant factor affecting positively the probability of a bank failure and its CAMEL rating downgrade (Berger \& De Young, 1997; Gilbert, Meyer \& Vaughan, 2000; Bassett, Lee \& Spiller 2015). In addition, NPLs affect significantly GDP level and other key macroeconomic factors and consequently

\footnotetext{
2 Turguttopbas (2018) analyses the procyclicality of Total Capital and Leverage ratios of the Turkish banking sector for the time period $2001-2015$ and indicates that (a) the Total Capital Ratio is procyclical in both normal periods and times of crisis, and (b) the Leverage Ratio is countercyclical during financial crisis periods. Pfeifer \& Pikhart (2019) examine the contribution of Leverage Ratio into capital regulation effectiveness using a sample data set of 14 selected European countries from 2007 to 2015 and find, among others, that this ratio is significantly lower procyclical compared to the Total Capital Ratio.

3 They mention that the Leverage Ratio could reduce the procyclicality problem, although with this ratio the link between capital charges and actual asset risk could vanish, suggesting further research on this topic.

4 Excluding $\mathrm{S}$ segment.
} 
the robustness of the economies and banks' financial health (Louzis, Vouldis \& Metaxas, 2012; Tanasković \& Jandrić, 2015; Anastasiou, Louri \& Tsionas, 2016). For management quality, the Operational Expenses to Operational Income ratio is used (Doumpos \& Zopounidis, 2010). The significance of earnings ability ratios is examined by Gopalan (2010) who highlights the importance of ROA and ROE as significant early warning indicators of banks' performance. The Total Loans to Total Deposits (L-t-D) ratio (inverse liquidity proxy) is an important measure of liquidity risk as well as a bank soundness and performance indicator (Van den End, 2013; Chiaramonte, Croci \& Poli, 2015). Bank size is used as a measure of the systemic importance of a bank (Bańbuła and Iwanicz-Drozdowska, 2016).

Four key variables are used - global bank, financial crisis and two interactions (crisis $\times$ global bank; crisis global bank $\times$ bank size) - and also country dummies.

Regarding the crisis variable, the cut-off point for financial crisis outburst is the last quarter of 2007; specifically, we define as pre-crisis and post-crisis period the time period before 2007Q4 and after 2007Q4, respectively. These cut-off dates are based on the timelines outlined by the Bank for International Settlements (BIS). The data set is not split into more sub-periods i.e. in pre-crisis, crisis and postcrisis phase, given that the duration of these phases and their effects on banks presumably were not uniform worldwide (Baur, 2012). Note that the expressions "post-crisis" and "during and after crisis" are used interchangeably, denoting essentially the same time period (i.e. from January 2008 to December 2015) although the expression "during and after crisis" refers to the time segment 20082009 which represents the peak of the global financial crisis (see BIS, 2009) and it is used as the appropriate time point for the interaction of global bank with crisis.

All variables' definitions are presented in Table 1. Summary statistics are reported in Table 2. 
Table 1: Variables

\begin{tabular}{|c|c|c|c|c|}
\hline CAMEL segment & Abbreviation & & Variable definition & Data source \\
\hline \multirow{2}{*}{$\begin{array}{l}\text { Capital } \\
\text { Adequacy }\end{array}$} & $L R$ & Leverage Ratio & Total Capital / Total Assets (\%) & DataStream \\
\hline & $\mathrm{TCR}$ & Total Capital Ratio & Total Capital / total risk-weighted assets (\%) & DataStream \\
\hline Asset quality & NPLS & NPLS & Non-Performing Loans / Total Loans (\%) & DataStream \\
\hline $\begin{array}{l}\text { Management } \\
\text { quality }\end{array}$ & $\begin{array}{l}\text { OPEXPENS } \\
\text { OPINCOME }\end{array}$ & $\begin{array}{l}\text { Operating Expenses to } \\
\text { Operating Income }\end{array}$ & Operating Expenses / Operating Income (\%) & $\begin{array}{l}\text { DataStream; } \\
\text { own estimations }\end{array}$ \\
\hline $\begin{array}{l}\text { Earning } \\
\text { ability }\end{array}$ & ROE & ROE & Return on Equity (\%) & DataStream \\
\hline \multirow[t]{4}{*}{ Liquidity } & LTD & $L-t-D$ & Total Loans / Total Deposits (\%) & DataStream \\
\hline & SIZE & Bank size & Log of Total Assets & $\begin{array}{l}\text { DataStream; } \\
\text { own estimations }\end{array}$ \\
\hline & GLOBAL & & 0 if non-global and 1 if global & \\
\hline & CRISIS & & 0 if time $\leq 200704$ and 1 if time $\geq 200801$ & \\
\hline
\end{tabular}

Note: Crisis cut-off dates are based on the timelines outlined by the BIS (2009).

Table 2: Summary statistics

\begin{tabular}{|c|c|c|c|c|c|c|c|c|c|}
\hline \multirow{2}{*}{ Variable } & \multicolumn{3}{|c|}{$\begin{array}{c}\text { All banks } \\
\text { (whole period) }\end{array}$} & \multicolumn{3}{|c|}{$\begin{array}{l}\text { Global banks } \\
\text { (whole period) }\end{array}$} & \multicolumn{3}{|c|}{$\begin{array}{l}\text { Non-global banks } \\
\text { (whole period) }\end{array}$} \\
\hline & Obs & Mean & $\begin{array}{l}\text { Standard } \\
\text { Deviation }\end{array}$ & Obs & Mean & $\begin{array}{l}\text { Standard } \\
\text { Deviation }\end{array}$ & Obs & Mean & $\begin{array}{l}\text { Standard } \\
\text { Deviation }\end{array}$ \\
\hline TCR & 1601 & 14.35 & 4.98 & 819 & 13.84 & 3.57 & 782 & 14.89 & 6.07 \\
\hline LR & 2214 & 18.81 & 13.16 & 996 & 16.80 & 9.19 & 1218 & 20.45 & 15.48 \\
\hline NPLS & 1955 & 3.40 & 5.89 & 945 & 3.12 & 5.17 & 1010 & 3.66 & 6.48 \\
\hline OPEXPENSOPINCOME & 2094 & 536.11 & 392.09 & 939 & 582.79 & 409.62 & 1155 & 498.15 & 373.13 \\
\hline $\mathrm{ROE}$ & 2337 & 11.16 & 32.66 & 1064 & 11.69 & 28.11 & 1273 & 10.72 & 36.04 \\
\hline LTD & 2288 & 117.66 & 57.16 & 1055 & 124.33 & 61.56 & 1233 & 111.96 & 52.47 \\
\hline SIZE & 2456 & 17.88 & 2.18 & 1097 & 19.31 & 1.80 & 1359 & 16.73 & 1.76 \\
\hline
\end{tabular}

Notes: 1. all variables are defined in Table 1. 2. 'Whole period' refers to December 1999 December 2015 (yearly observations).

\section{Methodology and hypotheses}

A bank is classified as global if it (a) operates in more than one continents via subsidiaries or branches, and (b) has an average asset size $\geq 100$ billion USD during the whole data period; the first criterion is a necessary one, while the second is not. These criteria are in line with Jeucken (2001), McCauley, McGuire \& von 
Peter (2010), Fillat, Garetto \& Götz (2013) ${ }^{5}$, De Haas \& Van Lelyveld (2014) ${ }^{6}$, and BCBS (2017). Also, the majority of the banks that are characterized as global are included in the FSB (2016) list of Global Systemically Important Banks (G-SIBs) and in the "Banks around the World" website ${ }^{7}$ for 2015-2016. The distribution of global and non-global banks per continent and country is depicted in Appendix A - Table A1.

For panel regressions the random effects model is employed because it allows for time invariant variables (Wooldridge, 2002) such as country of origin (headquarters). The general form of the panel data regression model can be written as (Eq.1):

$$
Y_{i t}=a+\beta X_{i t}+u_{i t}
$$

where, $i$ denotes entity and $t$ time, $\alpha$ is a scalar, $\beta$ is $\mathrm{K} \times 1, X_{i t}$ is the $i t$ th observation on K explanatory variables and $u_{i t}$ is the error term (Baltagi, 2005).

The employed panel data regression model in its combined form can be written as follows (Eq.2):

$$
Y_{i j t}=a+\beta X_{i j t-1}+\gamma D V_{s}+u_{i t}+\varepsilon_{i t}
$$

where, $i$ denotes entity (bank), $j$ country and $t$ time (year from 1999 to 2015), $Y_{i j t}$ is the dependent variable (Leverage Ratio, Total Capital Ratio) for bank $i$ in country $j$ in year $t, \alpha$ is the unknown intercept for each entity $i$ being estimated using random effects (RE), $X_{i j t-1}$ is a vector of lagged bank-level control variables (CAMEL factors ${ }^{8}$, bank size), and $D V_{s}$ is a vector of other control variables (including the key or main variables) expressed as dummy variables, and $u_{i t}$ and $\varepsilon_{i t}$ is the between-entity error and the within-error, respectively.

Moreover, we have chosen the clustering on the bank- rather than country-level, as some of the countries in the sample have more banks than others (see Appen$\operatorname{dix} A$ - Table 1A).

5 According to Fillat, Garetto \& Götz (2013) a foreign bank enters another country or market either by opening subsidiaries or via branches and agencies.

6 De Haas \& Van Lelyveld (2014) rank large banks worldwide according to their asset size and classify them as multinationals or global if they have more than one significant foreign bank subsidiary.

7 http://www.relbanks.com

8 Including lagged LR and TCR that are used interchangeably depending on the model. 
The model's fully-expanded version is described by Eq.3:

$Y_{i j t}=a+\beta_{i} X_{i j t-1}+\gamma_{1} C_{j}+\gamma_{2} C R_{t}+\gamma_{3} G L_{i j t}+\gamma_{4} I N T_{1}+\gamma_{5} I N T_{2}+u_{i t}+\varepsilon_{i t}(3)$

where, $C_{j}$ stands for country dummy, $C R_{t}$ is a dummy variable for crisis, $G L_{i j t}$ is a dummy variable for global bank, $I N T_{1}\left(C R_{t} \times G L_{i j t}\right)$ and $I N T_{2}\left(C R_{t} \times G L_{i j t} \times S I Z E_{i j t-1}\right)$ represent a two- and three-way interaction term respectively.

The purpose of adding the above-mentioned interactions is to test for significant differences between global and non-global banks during and after the financial crisis.

Four hypotheses are tested:

1. Ho: the crisis has not affected the Leverage Ratio concerning both banktypes; Ha: the crisis has affected the Leverage Ratio concerning both bank-types.

2. Ho: the crisis has not affected the Total Capital Ratio concerning both bank-types; Ha: the crisis has affected the Total Capital Ratio concerning both bank-types.

3. Ho: there are no differences in the Leverage and Total Capital ratios between global and non-global banks. Ha: there are differences in the Leverage and Total Capital ratios between global and non-global banks.

4. Ho: there are no differences between global and non-global banks during and after the financial crisis; Ha: there are differences between global and non-global banks during and after the financial crisis.

\section{Results}

Prior to regression analysis all variables were examined for unit roots, while a correlation analysis among the regressors was performed to avoid possible multicollinearity problems. The Augmented Dickey-Fuller Fisher-type and the Phillips Perron Fisher-type unit root test both reject the null hypothesis for the existence of unit roots (see Table 3). The correlation analysis indicates that all pairwise correlations coefficients are below $0.50^{9}$ (see Table 4).

\footnotetext{
9 This value can be considered as a threshold between comparatively low and moderate and upper correlations (Gujarati, 2004).
} 
Table 3: Panel unit root tests

\begin{tabular}{lcccc}
\hline & \multicolumn{2}{c}{ Fisher-type test: ADF } & \multicolumn{2}{c}{ Fisher-type test: $P P$} \\
& \multicolumn{2}{c}{ Inverse $\chi^{2} P$} & \multicolumn{2}{c}{ Inverse $\chi^{2} P$} \\
\hline Variable & Statistic & $p$-value & Statistic & $p$-value \\
\hline TR & 541.6484 & 0.0000 & 580.2269 & 0.0000 \\
\hline NPLS & 676.2576 & 0.0000 & 732.1591 & 0.0000 \\
\hline OPEXPENSOPINCOME & 906.8979 & 0.0000 & 661.8412 & 0.0000 \\
\hline ROE & 903.7929 & 0.0000 & 1455.9198 & 0.0000 \\
\hline LTD & 1332.9615 & 0.0000 & 1404.0071 & 0.0000 \\
\hline SIZE & 904.6114 & 0.0000 & 809.8842 & 0.0000 \\
\hline
\end{tabular}

Notes: 1. Fisher-type unit root test refers to the Phillips-Perron (PP) and AugmentedDickey-Fuller (ADF) tests, respectively; both tests were performed with constant term and no trend.

2. Ho: All panels contain unit roots; Ha: At least one panel is stationary.

Table 4: Correlation matrix

\begin{tabular}{lccccccc}
\hline Variable & TCR & LR & NPLS & $\begin{array}{l}\text { OPEXPENS } \\
\text { OPINCOME }\end{array}$ & ROE & LTD & SIZE \\
\hline TCR & 1.000 & & & & & & \\
\hline LR & 0.251 & 1.000 & & & & & \\
\hline NPLS & 0.158 & 0.042 & 1.000 & & & & \\
\hline OPEXPENSOPINCOME & -0.060 & 0.049 & 0.070 & 1.000 & & \\
\hline ROE & 0.010 & -0.100 & -0.142 & -0.121 & 1.000 & \\
\hline LTD & -0.130 & 0.494 & 0.032 & 0.208 & -0.042 & 1.000 & \\
\hline SIZE & -0.313 & -0.160 & -0.066 & 0.029 & -0.034 & 0.046 & 1.000 \\
\hline
\end{tabular}

Note: All variables are defined in Table 1.

Regressions results are reported in Tables 5 and 6. 
Table 5: Regressions results (LR)

\begin{tabular}{|c|c|c|c|c|c|c|c|c|c|}
\hline Dependent variable: $L R$ & (1) & $(2)$ & (3) & (4) & (5) & (6) & (7) & (8) & (9) \\
\hline \multicolumn{10}{|l|}{ Method of estimation: RE GLS } \\
\hline$T C R_{(T-1)}$ & & $\begin{array}{c}0.421^{* * *} \\
(5.22)\end{array}$ & $\begin{array}{r}-0.016 \\
(0.70)\end{array}$ & $\begin{array}{c}-0.029 \\
(1.08)\end{array}$ & $\begin{array}{l}0.016 \\
(0.43)\end{array}$ & $\begin{array}{l}0.018 \\
(0.50)\end{array}$ & $\begin{array}{l}0.019 \\
(0.53)\end{array}$ & $\begin{array}{l}0.019 \\
(0.52)\end{array}$ & $\begin{array}{l}0.024 \\
(0.61)\end{array}$ \\
\hline$L R_{(T-1)}$ & $\begin{array}{c}0.898^{* * *} \\
(30.65)\end{array}$ & & $\begin{array}{c}0.941^{* * *} \\
(32.33)\end{array}$ & $\begin{array}{c}0.939^{* * *} \\
(33.39)\end{array}$ & $\begin{array}{c}0.850^{* * *} \\
(20.28)\end{array}$ & $\begin{array}{c}0.850^{* * *} \\
(20.20)\end{array}$ & $\begin{array}{c}0.849^{* * *} \\
(20.14)\end{array}$ & $\begin{array}{c}0.849^{* * *} \\
(20.31)\end{array}$ & $\begin{array}{c}0.848^{* * *} \\
(20.24)\end{array}$ \\
\hline$N P L S_{(T-1)}$ & & $\begin{array}{c}-0.004 \\
(0.09)\end{array}$ & $\begin{array}{l}0.004 \\
(0.16)\end{array}$ & $\begin{array}{l}0.003 \\
(0.11)\end{array}$ & $\begin{array}{r}-0.008 \\
(0.30)\end{array}$ & $\begin{array}{r}-0.007 \\
(0.28)\end{array}$ & $\begin{array}{c}-0.008 \\
(0.31)\end{array}$ & $\begin{array}{r}-0.009 \\
(0.32)\end{array}$ & $\begin{array}{c}-0.008 \\
(0.32)\end{array}$ \\
\hline OPEXPENSOPINCOME $_{(T-1)}$ & & $\begin{array}{r}-0.001 \\
(0.88)\end{array}$ & $\begin{array}{c}0.0002 \\
(0.57)\end{array}$ & $\begin{array}{c}0.0002 \\
(0.59)\end{array}$ & $\begin{array}{c}0.0003 \\
(0.75)\end{array}$ & $\begin{array}{c}0.0003 \\
(0.78)\end{array}$ & $\begin{array}{c}0.0003 \\
(0.79)\end{array}$ & $\begin{array}{c}0.0003 \\
(0.78)\end{array}$ & $\begin{array}{c}0.0003 \\
(0.83)\end{array}$ \\
\hline $\mathrm{ROE}_{(\mathrm{T}-1)}$ & & $\begin{array}{c}0.020^{* *} \\
(2.31)\end{array}$ & $\begin{array}{c}0.036^{* * *} \\
(6.70)\end{array}$ & $\begin{array}{c}0.036^{* * *} \\
(6.79)\end{array}$ & $\begin{array}{c}0.035^{* * *} \\
(6.61)\end{array}$ & $\begin{array}{c}0.035^{* * *} \\
(6.91)\end{array}$ & $\begin{array}{c}0.036^{* * *} \\
(7.10)\end{array}$ & $\begin{array}{c}0.036^{* * *} \\
(7.06)\end{array}$ & $\begin{array}{c}0.036^{* * *} \\
(7.03)\end{array}$ \\
\hline $\operatorname{LTD}_{(\mathrm{T}-1)}$ & & $\begin{array}{l}0.033 \\
(1.58)\end{array}$ & $\begin{array}{l}0.005 \\
(1.12)\end{array}$ & $\begin{array}{l}0.005 \\
(1.18)\end{array}$ & $\begin{array}{r}-0.004 \\
(0.60)\end{array}$ & $\begin{array}{c}-0.004 \\
(0.60)\end{array}$ & $\begin{array}{c}-0.004 \\
(0.63)\end{array}$ & $\begin{array}{c}-0.004 \\
(0.63)\end{array}$ & $\begin{array}{c}-0.004 \\
(0.65)\end{array}$ \\
\hline $\operatorname{SIZE}_{(\mathrm{T}-1)}$ & & & & $\begin{array}{c}-0.116^{* *} \\
(1.99)\end{array}$ & $\begin{array}{l}-0.178 \\
(0.28)\end{array}$ & $\begin{array}{c}-0.010 \\
(0.16)\end{array}$ & $\begin{array}{l}0.066 \\
(0.78)\end{array}$ & $\begin{array}{l}0.067 \\
(0.81)\end{array}$ & $\begin{array}{l}0.098 \\
(0.95)\end{array}$ \\
\hline COUNTRY & & & & & YES & YES & YES & YES & YES \\
\hline CRISIS & & & & & & $\begin{array}{c}-0.090 \\
(0.32)\end{array}$ & $\begin{array}{l}-0.153 \\
(0.55)\end{array}$ & $\begin{array}{c}-0.244 \\
(0.57)\end{array}$ & $\begin{array}{l}-0.262 \\
(0.426)\end{array}$ \\
\hline GLOBAL & & & & & & & $\begin{array}{c}-0.399 \\
(1.33)\end{array}$ & $\begin{array}{r}-0.511 \\
(1.07)\end{array}$ & $\begin{array}{r}-0.587 \\
(1.20)\end{array}$ \\
\hline $\begin{array}{l}\text { INTERACTION1 } \\
(=\text { CRISIS } \times \text { GLOBAL })\end{array}$ & & & & & & & & $\begin{array}{l}0.158 \\
(0.29)\end{array}$ & $\begin{array}{l}2.413 \\
(0.82)\end{array}$ \\
\hline $\begin{array}{l}\text { INTERACTION2 } \\
(=C R I S I S \times G L O B A L \times S I Z E)\end{array}$ & & & & & & & & & $\begin{array}{l}-0.113 \\
(0.76)\end{array}$ \\
\hline Constant & $\begin{array}{c}2.077^{* * *} \\
(4.23) \\
\end{array}$ & $\begin{array}{c}7.990^{* * *} \\
(3.10) \\
\end{array}$ & $\begin{array}{l}0.493 \\
(1.14) \\
\end{array}$ & $\begin{array}{c}2.859^{* *} \\
(2.19) \\
\end{array}$ & $\begin{array}{c}3.154^{* *} \\
(2.24) \\
\end{array}$ & $\begin{array}{c}3.018^{* *} \\
(2.15) \\
\end{array}$ & $\begin{array}{l}1.752 \\
(1.04) \\
\end{array}$ & $\begin{array}{l}1.794 \\
(1.03) \\
\end{array}$ & $\begin{array}{l}1.210 \\
(0.57) \\
\end{array}$ \\
\hline Observations & 1994 & 1085 & 1049 & 1049 & 1049 & 1049 & 1049 & 1049 & 1049 \\
\hline No. of countries & - & - & - & - & 33 & 33 & 33 & 33 & 33 \\
\hline \multicolumn{10}{|l|}{ Diagnostics: } \\
\hline Rsquared & 0.838 & 0.217 & 0.816 & 0.817 & 0.828 & 0.828 & 0.828 & 0.828 & 0.828 \\
\hline Wald test: $p$-value & 0.0000 & 0.0000 & 0.0000 & 0.0000 & 0.0000 & 0.0000 & 0.0000 & 0.0000 & 0.0000 \\
\hline
\end{tabular}


Table 6: Regressions results (TCR)

\begin{tabular}{|c|c|c|c|c|c|c|c|c|c|}
\hline Dependent variable: TCR & (1) & $(2)$ & (3) & (4) & (5) & (6) & (7) & (8) & (9) \\
\hline \multicolumn{10}{|l|}{ Method of estimation: RE GLS } \\
\hline$T C R_{(T-1)}$ & $\begin{array}{c}0.835^{* * *} \\
(36.86)\end{array}$ & & $\begin{array}{c}0.815^{* * *} \\
(38.48)\end{array}$ & $\begin{array}{c}0.812^{* * *} \\
(30.29)\end{array}$ & $\begin{array}{c}0.743^{* * *} \\
(21.14)\end{array}$ & $\begin{array}{c}0.717^{* * *} \\
(24.38)\end{array}$ & $\begin{array}{c}0.716^{* * *} \\
(24.20)\end{array}$ & $\begin{array}{c}0.716^{* * *} \\
(24.61)\end{array}$ & $\begin{array}{c}0.704^{* * *} \\
(19.92)\end{array}$ \\
\hline$L R_{(T-1)}$ & & $\begin{array}{c}0.193^{* * *} \\
(3.89)\end{array}$ & $\begin{array}{c}0.027^{* * *} \\
(2.60)\end{array}$ & $\begin{array}{c}0.026^{* * *} \\
(2.71)\end{array}$ & $\begin{array}{c}0.046^{* *} \\
(2.34)\end{array}$ & $\begin{array}{c}0.046^{* *} \\
(2.30)\end{array}$ & $\begin{array}{c}0.046^{* * *} \\
(2.38)\end{array}$ & $\begin{array}{c}0.045^{* *} \\
(2.41)\end{array}$ & $\begin{array}{c}0.046^{* *} \\
(2.41)\end{array}$ \\
\hline$N P L S_{(T-1)}$ & & $\begin{array}{l}0.073^{*} \\
(1.86)\end{array}$ & $\begin{array}{l}0.013 \\
(0.92)\end{array}$ & $\begin{array}{l}0.013 \\
(0.87)\end{array}$ & $\begin{array}{l}0.022 \\
(1.24)\end{array}$ & $\begin{array}{l}0.016 \\
(0.90)\end{array}$ & $\begin{array}{l}0.017 \\
(0.94)\end{array}$ & $\begin{array}{l}0.016 \\
(0.88)\end{array}$ & $\begin{array}{l}0.016 \\
(0.90)\end{array}$ \\
\hline OPEXPENSOPINCOME $_{(\mathrm{T}-1)}$ & & $\begin{array}{c}-0.0001 \\
(0.33)\end{array}$ & $\begin{array}{l}0.0001 \\
(0.70)\end{array}$ & $\begin{array}{r}0.0001 \\
(0.69)\end{array}$ & $\begin{array}{c}0.0002 \\
(1.12)\end{array}$ & $\begin{array}{r}0.0001 \\
(0.51)\end{array}$ & $\begin{array}{l}0.0001 \\
(0.50)\end{array}$ & $\begin{array}{r}0.0001 \\
(0.45)\end{array}$ & $\begin{array}{c}0.0001 \\
(0.13)\end{array}$ \\
\hline $\mathrm{ROE}_{(\mathrm{T}-1)}$ & & $\begin{array}{l}-0.006 \\
(0.53)\end{array}$ & $\begin{array}{l}-0.002 \\
(0.97)\end{array}$ & $\begin{array}{l}-0.002 \\
(1.06)\end{array}$ & $\begin{array}{l}-0.003 \\
(1.22)\end{array}$ & $\begin{array}{c}0.0002 \\
(0.13)\end{array}$ & $\begin{array}{c}-0.0003 \\
(0,18)\end{array}$ & $\begin{array}{c}-0.0002 \\
(0.12)\end{array}$ & $\begin{array}{c}-0.0002 \\
(0.11)\end{array}$ \\
\hline $\operatorname{LTD}_{(\mathrm{T}-1)}$ & & $\begin{array}{c}-0.028^{* * *} \\
(2.77)\end{array}$ & $\begin{array}{c}-0.004^{* *} \\
(2.01)\end{array}$ & $\begin{array}{c}-0.004^{* *} \\
(2.05)\end{array}$ & $\begin{array}{c}-0.008^{* * *} \\
(2.87)\end{array}$ & $\begin{array}{c}-0.008^{* * *} \\
(3.28)\end{array}$ & $\begin{array}{c}-0.008^{* * *} \\
(3.30)\end{array}$ & $\begin{array}{c}-0.008^{* * *} \\
(3.45)\end{array}$ & $\begin{array}{c}-0.008^{* * *} \\
(3.40)\end{array}$ \\
\hline $\operatorname{SIZE}_{(T-1)}$ & & & & $\begin{array}{r}-0.031 \\
(0.52)\end{array}$ & $\begin{array}{l}-0.026 \\
(0.31)\end{array}$ & $\begin{array}{l}-0.119 \\
(1.40)\end{array}$ & $\begin{array}{c}-0.195^{* *} \\
(2.13)\end{array}$ & $\begin{array}{c}-0.189^{* *} \\
(2.10)\end{array}$ & $\begin{array}{c}-0.266^{* *} \\
(2.07)\end{array}$ \\
\hline COUNTRY & & & & & YES & YES & YEL & YES & YES \\
\hline CRISIS & & & & & & $\begin{array}{c}1.053^{* * *} \\
(7.74)\end{array}$ & $\begin{array}{c}1.113^{* * *} \\
(7.85)\end{array}$ & $\begin{array}{c}0.807^{* * *} \\
(2.66)\end{array}$ & $\begin{array}{c}0.858^{* * *} \\
(2.97)\end{array}$ \\
\hline GLOBAL & & & & & & & $\begin{array}{c}0.402^{* *} \\
(2.35)\end{array}$ & $\begin{array}{l}0.036 \\
(0.11)\end{array}$ & $\begin{array}{l}0.223 \\
(0.84)\end{array}$ \\
\hline $\begin{array}{l}\text { INTERACTION1 } \\
(=\text { CRISIS } \times \text { GLOBAL })\end{array}$ & & & & & & & & $\begin{array}{l}0.520 \\
(1.39)\end{array}$ & $\begin{array}{l}-5.305 \\
(1.45)\end{array}$ \\
\hline $\begin{array}{l}\text { INTERACTION2 } \\
(=\text { CRISIS } \times \text { GLOBAL } \times S I Z E)\end{array}$ & & & & & & & & & $\begin{array}{l}0.291 \\
(1.51)\end{array}$ \\
\hline Constant & $\begin{array}{c}2.573^{* * *} \\
(8.18)\end{array}$ & $\begin{array}{c}14.487^{* * *} \\
(15.03)\end{array}$ & $\begin{array}{c}2.647^{* * *} \\
(7.05)\end{array}$ & $\begin{array}{c}3.280^{* *} \\
(2.15)\end{array}$ & $\begin{array}{c}4.100^{* *} \\
(2.06)\end{array}$ & $\begin{array}{c}5.711^{* * *} \\
(3.05)\end{array}$ & $\begin{array}{c}6.976^{* * *} \\
(3.53)\end{array}$ & $\begin{array}{c}7.110^{* * *} \\
(3.47)\end{array}$ & $\begin{array}{c}8.572^{* * *} \\
(3.04)\end{array}$ \\
\hline Observations & 1427 & 1150 & 1065 & 1065 & 1065 & 1065 & 1065 & 1065 & 1065 \\
\hline No. of countries & - & - & - & - & 32 & 32 & 32 & 32 & 32 \\
\hline \multicolumn{10}{|l|}{ Diagnostics: } \\
\hline Rsquared & 0.728 & 0.162 & 0.741 & 0.741 & 0.755 & 0.763 & 0.764 & 0.764 & 0.766 \\
\hline Wald test: $p$-value & 0.0000 & 0.0000 & 0.0000 & 0.0000 & 0.0000 & 0.0000 & 0.0000 & 0.0000 & 0.0000 \\
\hline
\end{tabular}

Notes to Tables 5 and 6: 1. Group variable: bank. 2. Absolute values in parentheses denote heteroskedasticity-robust z-statistics. 3. ${ }^{* * *}$ significant at the $1 \%$ level; **significant at the $5 \%$ level; * significant at the $10 \%$ level. 4 . YES denotes that countries dummies are included. 5 . In specifications $5-9$, five (Table 5) and six (Table 6) countries were omitted because of collinearity.

As for the first hypothesis, we accept Ho i.e. the crisis has no impact on LR of both bank types (crisis estimate in statistically insignificant - see Table 5); this result supports prior relevant findings by Smith, Grill \& Lang $(2017)^{10}$ for the

${ }^{10}$ They use a panel data set of 500 EU banks over the period 2005-2014 and show, inter alia, that the traditional Leverage Ratio is an important tool for supervisory purposes and banks comparison in terms of risk taking. 
European banking sector. Regarding second hypothesis, we reject Ho and we accept Ha i.e. the crisis has a positive effect on TCR of both bank types (crisis estimate is significant at the $1 \%$ level - see Table 6) presumably as a result of capital injections by the State. These findings further imply that, irrespective of bank type, the LR is not sensitive to financial crisis, but TCR is crisis sensitive.

Considering third hypothesis we accept $\mathrm{Ho}$ and we reject $\mathrm{Ha}$ as our results show that global banks do not perform better (or worse) than their non-global counterparts in terms of these ratios during the whole data period as global bank estimate is statistically insignificant in both cases (see Tables 5 and 6); note that GLOBAL estimate becomes insignificant when a two-way interaction (crisis $\times$ global bank) is introduced

Finally, as for the fourth hypothesis, we accept Ho and we reject Ha since the two-way interaction term (crisis $\times$ global bank) is not statistical significant. This finding shows that global banks do not exhibit a statistically different LR or TCR relative to non-global banks, during and after the financial crisis; this also holds when a three-way interaction (crisis $\times$ global bank $\times$ bank size) is introduced (see Table 5 and Table 6).

As for the rest of the control variables, it is shown that:

(a) LR exercises a positive impact on TCR regardless of bank type and period (see Table 6); however, when the dependent variable is the LR, the TCR is statistically insignificant (see Table 5);

(b) NPLS has no effect on both the LR and TCR (see Tables 5 and 6), thus showing that credit risk does not exert an impact on both ratios (at least not a direct one), whether a bank is global or not and irrespective of time period, a result that may be attributed to various reasons, as for example the effect of NPLs in one or more continent/s (e.g. Europe) is absorbed by the effect of NPLs in another continent/s (e.g. North America, Oceania) and/or to the different regulatory treatment of Non-performing exposures (NPEs) and NPLs across different countries and jurisdictions;

(c) LTD has no effect on the LR, but it has a statistically significant negative impact on the TCR i.e. liquidity risk does not have an impact on the LR (see Table 5) but has a negative one on the TCR (see Table 6), whether a bank is global or not and irrespective of time period;

(d) ROE has a statistically significant (and positive) effect on the LR only, further implying that this ratio is affected by profitability only whether a bank is global or not, during the whole data period (see Table 5); 
(e) Bank size has no effect on the LR regardless of bank type (size effect becomes insignificant after country inclusion) (see Table 5), although it has a statistically significant negative impact on the TCR after GLOBAL variable introduction (note that this effect vanishes when a two - and three-way interaction, respectively, is introduced - see Table 6).

\section{Conclusions}

This paper compares global and non-global banks concerning Leverage and Total Capital Ratio, covering both normal periods and times of crisis. It is shown that (a) the crisis has positively affected only the Total Capital Ratio of both banktypes, (b) global banks do not exhibit differential behaviour in terms of both ratios in both the pre- and post-crisis periods, and (c) global banks do not exhibit superior or inferior performance relative to their non-global counterparts during and after the recent financial crisis. As for the other control variables used, the most notable findings are that (a) bank earning ability (as expressed by ROE ratio) has a positive impact on the Leverage Ratio only during the whole data period and regardless of bank type, and (b) credit risk (as expressed by NPLs ratio) does not have an impact (at least not a direct one) on both Leverage and Total Capital ratios, whether a bank is global or not and irrespective of time period, a result that may be ascribed to various reasons such for instance as the different regulatory regimes for NPLs and NPEs worldwide.

Our findings may be of interest to supervising authorities and bank regulators since it is suggests, inter alia, that the Leverage Ratio is perhaps a more appropriate tool for capital adequacy measurement purposes - especially for global banks - regardless of crisis or non-crisis periods. Finally, on the basis of our results, a topic for future research could be the investigation of the possibility that global banks may have not contributed to the dissemination of the 2007-2008 financial crisis: other transmission channels may be more relevant, such as through countries (a sovereign debt crisis evolves into a banking crisis and expands among countries through global banks interconnectedness - see e.g. Mody \& Sandri (2012) - while, as Laeven \& Valencia (2012) mention, many banking crisis occur together with currency or sovereign debt crises (e.g. Greece). 


\section{References}

1. Anastasiou, D., Louri, H., and Tsionas, M. (2016). "Determinants of nonperforming loans: Evidence from Euro area countries". Finance Research Letters, 18, 116-119.

2. Altunbas, Y., Manganelli, S. and Marques-Ibanez, D. (2011). "Bank risk during the financial crisis: do business models matter?” European Central Bank, Working Paper No. 1394.

3. Baltagi, H.D. (2005). Econometric Analysis of Panel Data, $3^{\text {rd }}$ ed., John Wiley \& Sons Ltd.

4. Bank for International Settlements (BIS) (2009). 79 ${ }^{\text {th }}$ Annual Report, 29 June 2009. Basel: BIS.

5. Banks around the World (2016). “Top Banks in the World”, update: June 10, 2016 (https://www.relbanks.com/worlds-top-banks/assets, accessed: 23 July 2016).

6. Bańbuła, P. and Iwanicz-Drozdowska, M. (2016). “The systemic importance of banks - name and shame seems to work". Finance Research Letters, 18, 297-301

7. Basel Committee on Banking Supervision (BCBS) (2013). Global systemically important banks: assessment methodology and the higher loss absorbency requirement, July 2013. Basel: Bank for International Settlements (BIS).

8. Basel Committee on Banking Supervision (BCBS) (2017). Global systemically important bank - revised assessment framework", March 2017. Basel: Bank for International Settlements (BIS).

9. Basel Committee on Banking Supervision (BCBS) (2019). Leverage ratio requirements for global systemically important banks. Bank for International Settlements (BIS). Retrieved from https://www.bis.org/basel framework/ chapter/LEV/40.htm?inforce=20220101 (2019, August 24).

10. Bassett, F.W., Lee, J. S. and Spiller, P. T. (2015). "Estimating changes in supervisory standards and their economic effects". Journal of Banking \& Finance, 60, 21-43.

11. Baur, G.D. (2012). "Financial contagion and the real economy". Journal of Banking \& Finance, 36, 2680-2692.

12. Beck, T., Demirgüc-Kunt, A. and Merrouche, O. (2013). "Islamic vs. conventional banking: Business model, efficiency and stability". Journal of Banking \& Finance, 37, 433-447.

13. Behn, M., Haselmann, R. and Wachtel, P. (2016). "Procyclical Capital Regulation and Lending”. Journal of Finance, Vol. LXXI-No.2, 919-955.

14. Berger, N.A. and DeYoung, R. (1997). "Problem loans and cost efficiency in commercial banks”. Journal of Banking \& Finance, 21, 849-870. 
15. Cetorelli, N. And Goldberg, S.L. (2010). "Global Banks and International Shock Transmission: Evidence from the Crisis", Federal Reserve Bank of New York Staff Reports, No.446, May 2010.

16. Chiaramonte, L., Croci, E. and Poli, F. (2015), "Should we trust the Z-score? Evidence from the European Banking Industry". Global Finance Journal, 28, 111-131.

17. De Haan, L., and J. Kakes. (2019). "European banks after the global financial crisis: peak accumulated losses, twin crises and business models", Journal of Banking Regulation. https://doi.org/10.1057/s41261-019-00107-y .

18. De Haas, R. and Van Lelyveld, I. (2014), "Multinational Banks and the Global Financial Crisis: Weathering the Perfect Storm?”. Journal of Money, Credit and Banking, 46 (1), 333-364.

19. Doumpos, M. and Zopounidis, C. (2010), "A multicriteria decision support system for bank rating". Decision Support Systems, 50, 55-63.

20. European Banking Authority (EBA) (2016). CRD IV - CRR/Basel III Monitoring Exercise - Results based on data as of 30 June 2015, 2 March 2016, EBA.

21. Fillat L.J., Garetto, S. and Götz M. (2013). "Multinational Banks", December 7, 2013. Retrieved from https://cepr.org/sites/default/files/Fillat.pdf (2018, March 23).

22. Financial Stability Board (FSB) (2016). 2016 list of global systemically important banks (G-SIBs). FSB

23. Francis, B.W. (2014). "UK deposit-taker responses to the financial crisis: what are the lessons?", Bank of England, Working Paper 501 - June 2014.

24. Gilbert, A.R., Meyer, P.A. and Vaughan, D.M. (2000). "The Role of a CAMEL Downgrade Model in Bank Surveillance”, Federal Reserve Bank of St. Louis, Working Paper, 2000-021A.

25. Gopalan, K.Y. (2010). "Earliest Indicator of Bank Failure Is Deterioration in Earnings”, Federal Reserve Bank of St. Louis, Central Banker, Spring 2010.

26. Gujarati, D. (2004). Basic Econometrics, fourth ed., New York: McGraw-Hill Companies.

27. Hale, G., Kapan, T. and Minoiu, C. (2016). "Crisis Transmission through the Global Banking Network”, International Monetary Fund (IMF), Working Paper WP/16/91.

28. Hogan, L.T. (2015). "Capital and risk in commercial banking: A comparison of capital and risk-based capital ratios". The Quarterly Review of Economics and Finance, 57, 32-45.

29. Jeucken, M. (2001). Sustainable Finance and Banking: The Financial Sector and the Future of the Planet, Earthscan Puplications Ltd, London.

30. Kalemli-Ozcan, S., Papaioannou, E. and Perri, F. (2013). "Global banks and crisis transmission”. Journal of International Economics, 89, 495-510. 
31. Laeven, L., and Valencia, F. (2012). "Systemic Banking Crises Database: An Update”, International Monetary Fund (IMF). IMF Working Paper WP/12/163 - June 2012.

32. Liu, X.E. and Pogach, J. (2017). “The effect of foreign lending on domestic loans: An analysis of US global banks”. Economic Letters, 156, 151-154.

33. Louzis, D., Vouldis, A., and Metaxas, V. (2012). "Macroeconomic and bankspecific determinants of non-performing loans in Greece: A comparative study of mortgage, business and consumer loan portfolios". Journal of Banking \& Finance, 36 (4), 1012-1027.

34. McCauley, N.R., Ruud, S.J. and Wooldridge, D.P. (2002). "Globalising international banking". BIS Quarterly Review, March 2002, 41-51.

35. McCauley, R., McGuire, P. and von Peter, G. (2010). "The architecture of global banking: from international to multinational”. BIS Quarterly Review, March 2010, 25-37.

36. Mody, A. and Sandri, D. (2012). "The eurozone crisis: how banks and sovereigns came to be joined at the hip". Economic Policy, 27 (70), 199-230.

37. Moosa, I. (2010). "The myth of too big to fail”. Journal of Banking Regulation, 11, 319-333.

38. Niepmann, F. (2011). "Banking across Borders”, De Nederlandsche Bank (DNB), Working Paper 325 - December 2011.

39. Pfeifer, L. and Pikhart, Z. (2019). "Leverage Ratio and its Potential For Enhancing the Effectiveness of Capital Regulation”. Journal of Central Banking Theory and Practice, 8 (2), 129-146.

40. Smith, A.J., Grill, M. and Lang, J.H. (2017). “The leverage ratio, risk-taking and bank stability”, European Central Bank, Working Paper 2079.

41. Tanasković, S. and Jandrić, M. (2015). "Macroeconomic and Institutional Determinants of Non-performing Loans". Journal of Central Banking Theory and Practice, 4 (1), 47-62.

42. Turguttopbas, N.T. (2018). "Are Capital Ratios Procyclical? Evidence from Turkish Banking Data". Journal of Central Banking Theory and Practice, 7 (3), 159-180.

43. Van den End, W.J. (2013). "A macroprudential approach to address liquidity risk with the Loan-to-Deposit ratio", De Nederlandsche Bank (DNB), Working Paper 372 - February 2013.

44. Yuksel, M. (2014). "Identifying Global Systemically Important Financial Institutions”, Reserve Bank of Australia, Bulletin, December Quarter 2014, 63-72.

45. Wooldridge, M.J. (2002). Econometric Analysis of Cross Section and Panel Data. Cambridge, Massachusetts, London, England: The MIT Press. 


\section{Appendix A}

Table A1: Distribution of global and non-global banks per continent and country

\begin{tabular}{|c|c|c|c|c|}
\hline Continent & Country & All banks & Global banks & Non-global banks \\
\hline \multirow{6}{*}{ Africa } & Kenya & 2 & 0 & 2 \\
\hline & Mauritius & 2 & 1 & 1 \\
\hline & Morocco & 2 & 0 & 2 \\
\hline & Nigeria & 12 & 6 & 6 \\
\hline & South Africa & 8 & 4 & 4 \\
\hline & Total & 26 & 11 & 15 \\
\hline \multirow[t]{2}{*}{ Asia } & India & 2 & 0 & 2 \\
\hline & Total & 2 & 0 & 2 \\
\hline \multirow{9}{*}{ Asia Pacific } & Bangladesh & 2 & 0 & 2 \\
\hline & China & 8 & 4 & 4 \\
\hline & Indonesia & 2 & 0 & 2 \\
\hline & Japan & 10 & 4 & 6 \\
\hline & Malaysia & 2 & 1 & 1 \\
\hline & South Korea & 6 & 3 & 3 \\
\hline & Taiwan R.O.C. & 2 & 0 & 2 \\
\hline & Thailand & 2 & 0 & 2 \\
\hline & Total & 34 & 12 & 22 \\
\hline \multirow{14}{*}{ Europe } & Belgium & 2 & 1 & 1 \\
\hline & Denmark & 2 & 0 & 2 \\
\hline & France & 6 & 5 & 1 \\
\hline & Germany & 4 & 3 & 1 \\
\hline & Greece & 2 & 0 & 2 \\
\hline & Italy & 4 & 3 & 1 \\
\hline & Netherlands & 3 & 2 & 1 \\
\hline & Norway & 2 & 1 & 1 \\
\hline & Spain & 8 & 5 & 3 \\
\hline & Sweden & 4 & 4 & 0 \\
\hline & Switzerland & 6 & 4 & 2 \\
\hline & Turkey & 6 & 0 & 6 \\
\hline & UK & 5 & 5 & 0 \\
\hline & Total & 54 & 33 & 21 \\
\hline
\end{tabular}


Table A1: Distribution of global and non-global banks per continent and country (continued)

\begin{tabular}{|c|c|c|c|c|}
\hline Continent & Country & All banks & Global banks & Non-global banks \\
\hline \multirow{8}{*}{ Latin America } & Argentina & 2 & 0 & 2 \\
\hline & Brazil & 6 & 3 & 3 \\
\hline & Chile & 2 & 0 & 2 \\
\hline & Colombia & 2 & 0 & 2 \\
\hline & Ecuador & 2 & 0 & 2 \\
\hline & Mexico & 2 & 0 & 2 \\
\hline & Peru & 2 & 0 & 2 \\
\hline & Total & 18 & 3 & 15 \\
\hline \multirow[t]{2}{*}{ Middle East } & Oman & 2 & 0 & 2 \\
\hline & Total & 2 & 0 & 2 \\
\hline \multirow{3}{*}{ North America } & Canada & 10 & 5 & 5 \\
\hline & USA & 12 & 3 & 9 \\
\hline & Total & 22 & 8 & 14 \\
\hline \multirow[t]{3}{*}{ Oceania } & Australia & 7 & 4 & 3 \\
\hline & Total & 7 & 4 & 3 \\
\hline & Grand total & 165 & 71 & 94 \\
\hline
\end{tabular}

Notes: Data source: FSB (2016); Banks around the World (2016) (http://www.relbanks.com); own research. 